Check for updates

Cite this: Mater. Horiz., 2018, 5,536

Received 19th February 2018, Accepted 28th March 2018

DOI: $10.1039 / \mathrm{c} 8 \mathrm{mh} 00216 \mathrm{a}$

rsc.li/materials-horizons

\section{Three-dimensional strain engineering in epitaxial vertically aligned nanocomposite thin films with tunable magnetotransport properties $\uparrow$}

\author{
Xing Sun, ${ }^{a}$ Jijie Huang, ${ }^{a}$ Jie Jian, ${ }^{a}$ Meng Fan, ${ }^{a}$ Han Wang, ${ }^{a}$ Qiang Li, ${ }^{a}$ \\ Judith L. Mac Manus-Driscoll, ${ }^{b}$ Ping Lu, ${ }^{c}$ Xinghang Zhang ${ }^{a}$ and Haiyan Wang (D) *a
}

\begin{abstract}
Three-dimensional (3D) frameworks have been successfully constructed by interlayering $\mathrm{La}_{0.7} \mathrm{Sr}_{0.3} \mathrm{MnO}_{3}$ (LSMO) $-\mathrm{CeO}_{2}$ based epitaxial vertically aligned nanocomposite (VAN) thin films with pure $\mathrm{CeO}_{2}$ (or LSMO) layers. Such 3D interconnected $\mathrm{CeO}_{2}$ scaffolds integrate the lateral film strain by the interlayers with the vertical strain in VAN layers, and thus achieve the maximized strain tuning in LSMO. More importantly, by varying the types of the interlayers (i.e., $\mathrm{CeO}_{2}$ or $\mathrm{LSMO}$ ) and the number of interlayers from 1 to 3 layers, such 3D framework nanostructures effectively tune the electrical transport properties of LSMO, e.g., from a $3 \mathrm{D}$ insulating $\mathrm{CeO}_{2}$ framework with integrated magnetic tunnel junction structures, to a 3D conducting LSMO framework, where the magnetoresistance (MR) peak values have been tuned systematically to a record high of $66 \%$ at $56 \mathrm{~K}$ and enhanced MR properties at high temperatures above room temperature ( $\sim 325 \mathrm{~K}$ ). This new 3D framed design provides a novel approach in maximizing film strain, enhancing strain-driven functionalities, and manipulating the electrical transport properties effectively.
\end{abstract}

\section{Introduction}

$\mathrm{ABO}_{3}$-Based perovskites have attracted substantial research interest and have been widely applied in various modern electronic devices, because of their versatile electrical and magnetic properties, such as superconductivity, colossal magnetoresistance (CMR), ferroelectricity, ferromagnet, and multiferroicity. ${ }^{1-8}$ The physical properties of perovskite oxides are mainly determined by their building blocks- $\mathrm{BO}_{6}$ octahedral units. Constructed by the B-site transition metal cation coordinating with six oxygen ligands, $\mathrm{BO}_{6}$ octahedral units interconnect with each other by sharing corners and forming a three-dimensional (3D) framework. Altering the

\footnotetext{
${ }^{a}$ School of Materials Engineering, Purdue University, West Lafayette, IN 47907 , USA. E-mail: hwangoo@purdue.edu

${ }^{b}$ Department of Materials Science and Metallurgy, University of Cambridge, Cambridge, CB3 OFS, UK

${ }^{c}$ Sandia National Laboratories, Albuquerque, NM 87185, USA

$\dagger$ Electronic supplementary information (ESI) available. See DOI: 10.1039/c8mh00216a
}

\section{Conceptual insights}

We present a novel concept that implements a three dimensional (3D) strain scheme in epitaxial thin films. This is achieved by combining 2-phase vertically aligned nanocomposite with thin interlayers to effectively couple vertical interface strain with the lateral interface strain. Different from conventional epitaxy thin films and the vertically aligned nanocomposite (VAN) thin films, the 3D strain scheme takes advantages of both architectures and promotes various strain-driven physical properties, e.g., record high magnetoresistance values (MR\%) as low field magnetoresistance properties have been demonstrated in the $\mathrm{La}_{0.7} \mathrm{Sr}_{0.3} \mathrm{MnO}_{3}$ ( $\mathrm{LSMO}$ )- $\mathrm{CeO}_{2}$ interlayered with different numbers of $\mathrm{CeO}_{2}$ interlayers. This work brings a new approach to achieve highly strained films beyond the critical thickness in epitaxy thin films and to demonstrate enhanced vertical strain coupling by the 3D strain scheme. This demonstration not only shows the power of 3D strain scheme in strain engineering, multifunctionality coupling, and flexibility in structural designs, but also fulfills the urgent demands of new material designs for future electronic devices.

size, shape, and connectivity of those units can directly tune the B-O-B bonds, crystal structure, and other physical characteristics, such as magnetic anisotropy, transition temperature, electronic bandgaps, thermal conductivity, and magnetotransport properties. ${ }^{9-12}$ Strain engineering has been agreed on as one of the most effective approaches for such structural and property tuning. ${ }^{10-13}$

Great effort has been devoted to exploring effective strain engineering. One of the most effective approaches is substrate strain control where various substrates with different lattice parameters are explored for in-plane strain control. ${ }^{14-16}$ The approach provides an effective lateral strain tuning in relatively thin films (within the critical thickness of a few nanometers) since the substrate strain is relieved with increasing film thickness. ${ }^{17,18}$ In parallel, implementing heteroepitaxial 2-phase nanocomposite thin films can incorporate additional interfacial strain into the matrix film through interface coupling between the two phases, such as zero-dimensional (OD) nanoparticles in matrix, two-dimensional (2D) multilayered films, and very recently, vertically aligned nanocomposite (VAN) thin films. ${ }^{18-29}$ 

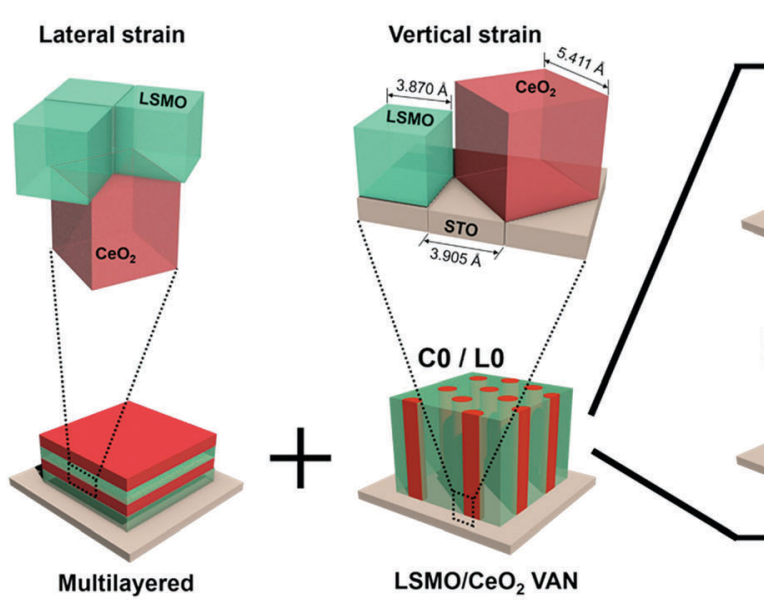

3-D $\mathrm{CeO}_{2}$ framework

Fig. 1 Schematic illustration of 2-phase heterogeneous microstructure evolution of the thin films: from vertical aligned nanocomposite (VAN) CO/LO to 3D $\mathrm{CeO}_{2}$ framed thin films $\mathrm{C} 1-\mathrm{C} 3$ and 3D LSMO framed thin films L1-L3. The 3D framed microstructure is achieved by alternative growth of the single phase and the VANs in multilayered fashion. This design combines the lateral strain introduced from multilayered thin film and the vertical strain from interfacial coupling in VANs, creates 3D interconnected $\mathrm{CeO}_{2}$ or $\mathrm{LSMO}$ framework microstructures within the thin films, and provides a versatile tool to achieve 3D strain tuning. The unit cells and phase of LSMO are in green, and the unit cells and phase of $\mathrm{CeO}_{2}$ are in red.

In VANs, the lattice strain can be maintained in much thicker films because of the vertical interface coupling. In addition, the density of the secondary phase, column dimensions and morphologies are also considered as major factors in the strain tuning of VAN films. Using VANs, a broad range of multifunctionalities, such as multiferroicity, ferroelectricity, low field magnetoresistance (LFMR), and anisotropic electrical/ionic transport properties have been demonstrated. ${ }^{11,17,20,22,25,30}$

3D nanostructured materials such as branched nanorods or nanoforests have attracted extensive research attentions because of their unique 3D nature. Taking full advantages of the vertical and horizontal dimensions, these 3D nanostructures exhibit many fascinating physical and chemical properties because of the highly enhanced interfacial area and stability, compared to the one-dimensional (1D) nanowire arrays. ${ }^{31-35}$ However, such 3D nanostructure designs in epitaxial thin film forms are scarce. To take advantages of the above strain control approaches and to explore the ultimate strain control in thin films, in this work, we propose a 3D strain architecture to combine the lateral strain introduced by the multilayered interfaces and the vertical strain from the vertical interface in VANs (as illustrated in Fig. 1). More specifically, this 3D strain control is achieved by the alternative growth of the single phase and the VANs in a multilayered fashion. $\mathrm{La}_{0.7} \mathrm{Sr}_{0.3} \mathrm{MnO}_{3}$ (LSMO) is selected as the matrix for this 3D strain design as it has shown a strong CMR effect, magnetic anisotropy, spin-glass like behavior, and LFMR properties. ${ }^{11,22,36,37}$ Various secondary phases have been demonstrated for enhanced LFMR in LSMO VAN films. ${ }^{19,36-39} \mathrm{CeO}_{2}$ is selected as the insulating secondary phase embedded in the LSMO matrix, because of its good in-plane lattice match with LSMO and STO after a $45^{\circ}$ in-plane rotation (Fig. 1), as well as its high thermal/mechanical stability. ${ }^{26,36,38}$ Furthermore, $\mathrm{CeO}_{2}$ can serve as the insulating layer favoring spin-dependent tunneling, and is easier to explore the synergistic effects between strain tuning and the LFMR effect.
As illustrated in the left panel of Fig. 1, either $\mathrm{CeO}_{2}$ or LSMO single layer is selected to construct the $3 \mathrm{D} \mathrm{CeO}_{2}$ framework or the 3D LSMO framework, respectively. More specifically, the single layer $\mathrm{LSMO}^{-\mathrm{CeO}_{2}}$ (molar ratio of $\mathrm{LSMO}: \mathrm{CeO}_{2}=1: 1$, also called L5C5) VAN thin films were prepared as control experiments and named as $\mathrm{C} 0$ or $\mathrm{L} 0$, without $\mathrm{LSMO}$ or $\mathrm{CeO}_{2}$ as the interlayers. $3 \mathrm{D} \mathrm{CeO}_{2}$ interlayered thin films with 1,2 , and 3 interlayers inserted in VAN structures are named as samples C1, C2, and C3, respectively. Similarly, 3D LSMO interlayered thin films with 1,2 , and 3 interlayers inserted in VAN are named as sample L1, L2, and L3, respectively. To explore the power of 3D strain effects offered by the 3D framework built in the $\mathrm{LSMO}-\mathrm{CeO}_{2}$ VAN systems, the molar ratio of LSMO and $\mathrm{CeO}_{2}$ is maintained at 1:1 in the VAN layers. Such ratio was selected based on the previous reports where effective tunneling effects from the secondary phase have been found..$^{27,36,37,40}$ This set of interlayered samples are expected to combine the vertical strain control by the VAN structures and the lateral strain control by the interlayers to achieve 3D strain modulation. LFMR properties are measured and correlated with the strain in these 3D strained frameworks.

\section{Results and discussion}

Transmission electron microscopy (TEM) and scanning transmission electron microscopy (STEM) images (Fig. 2) confirm the microstructures of samples $\mathrm{C} 0-\mathrm{C} 3$ and $\mathrm{L} 0-\mathrm{L} 3$. The thickness of all the as-deposited films are around 100-120 nm. A columnar nanocomposite structure with the alternative columns of LSMO and $\mathrm{CeO}_{2}$ can be clearly seen in the VAN thin film $\mathrm{C} 0$ from the cross-sectional TEM and the corresponding STEM images (Fig. 2a and b), respectively. It is obvious that the $\mathrm{CeO}_{2}$ nanopillars with a large aspect ratio are vertically grown in the LSMO matrix as seen from the high resolution TEM (HRTEM) image in Fig. 2c. 

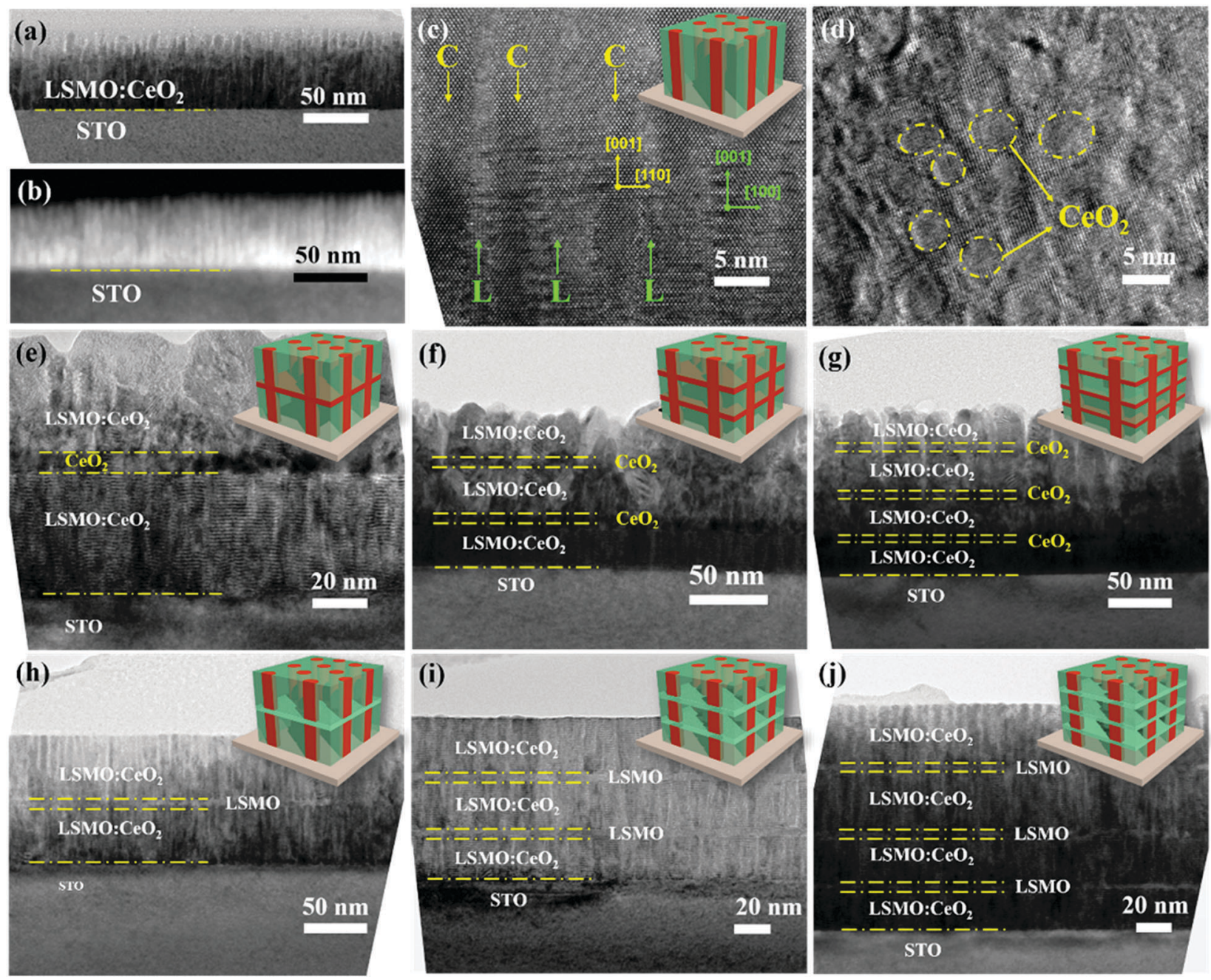

Fig. 2 (a) Cross-sectional TEM image of the VAN thin film CO and (b) its corresponding STEM image at low magnification. (c) Cross-sectional and (d) plan-view HRTEM images of sample CO. In the HRTEM image of (c), " $\mathrm{C}$ " in yellow points out the $\mathrm{CeO}_{2}$ nanopillars and "L" in green points out the LSMO matrix. Clearly, those $\mathrm{CeO}_{2}$ nanopillars with a large aspect ratio are vertically aligned and well distributed in the LSMO matrix and the sharp phase boundaries suggest the well separated growth of the two phases. Cross-sectional TEM images of the thin films (e-g) C1-C3 and ( $h-j) L 1-L 3$, showing the microstructures of 3D interconnected $\mathrm{CeO}_{2}$ and LSMO frames embedded within the thin films respectively.

The uniform distribution of the $\mathrm{CeO}_{2}$ nanopillars in-plane is confirmed by the plan-view TEM image of C0 (Fig. 2d and Fig. S1f, ESI $\dagger$ ). These images present clear and sharp interfaces between the $\mathrm{LSMO} / \mathrm{CeO}_{2}$ phases with limited inter-diffusion. In addition, the diameter of the circular-shaped $\mathrm{CeO}_{2}$ nanopillar domains is estimated to be $2-5 \mathrm{~nm}$. Fig. $2 \mathrm{e}-\mathrm{g}$ and $\mathrm{h}-\mathrm{j}$ present the 3D framed structured samples C1-C3 and L1-L3, respectively. Overall the structures are all grown as the designed 3D framed structures, as shown in the inset. For example, in the $3 \mathrm{D} \mathrm{CeO}_{2}$ framed thin films $\mathrm{C} 1-\mathrm{C} 3$, each $\mathrm{CeO}_{2}$ interlayer is around $5 \mathrm{~nm}$ thick, sandwiched between $\mathrm{LSMO}-\mathrm{CeO}_{2}$ VAN layers, and joined by vertical $\mathrm{CeO}_{2}$ nanopillars in the VAN layers to form a $3 \mathrm{D} \mathrm{CeO}_{2}$ frame embedded in the thin films. Similarly, in the 3D LSMO framed thin films L1-L3, each LSMO interlayer is around $5 \mathrm{~nm}$ thick and sandwiched between the $\mathrm{LSMO}-\mathrm{CeO}_{2}$ VAN layers. It is noted that as the number of $\mathrm{CeO}_{2}$ interlayers increases, the surface roughness increases gradually from C1 to C3. This might be related to the surface roughness introduced by $\mathrm{CeO}_{2}$ interlayers. In contrast, the incorporation of LSMO interlayers maintains relatively smooth film surfaces in L1-L3 without obvious porosity on the top surfaces (Fig. $2 \mathrm{~h}-\mathrm{j}$ ). Instead, a well-defined 3D interconnected LSMO frame is clearly observed within the dense films. Overall these VAN thin films are grown with a high epitaxial quality despite the introduced interlayers. The film quality is comparable with previously reported LSMO-based nanocomposites. ${ }^{36,38}$

XRD was employed to study the evolution of the out-of-plane strain in both $\mathrm{CeO}_{2}$ and LSMO phases as a function of the interlayer structure. The full XRD $2 \theta-\omega$ patterns of samples $\mathrm{C} 0-\mathrm{C} 3$ are present in Fig. 3. The $(00 l)$ diffraction peaks for both are present which confirms that $\mathrm{CeO}_{2}$ and LSMO have grown highly textured along the (00l) direction on $\mathrm{STO}(001)$ substrates, despite the minor LSMO(111) texture in the films. To reveal the strain state of $\mathrm{LSMO}$ and $\mathrm{CeO}_{2}$, the local scans of $\mathrm{CeO}_{2}(004)$ and $\mathrm{LSMO}(003)$ are plotted in Fig. $3 \mathrm{~b}$ and $\mathrm{c}$ for all the $\mathrm{C} 0-\mathrm{C} 3$ samples. It is clear that, as the number of $\mathrm{CeO}_{2}$ interlayer increases, the 

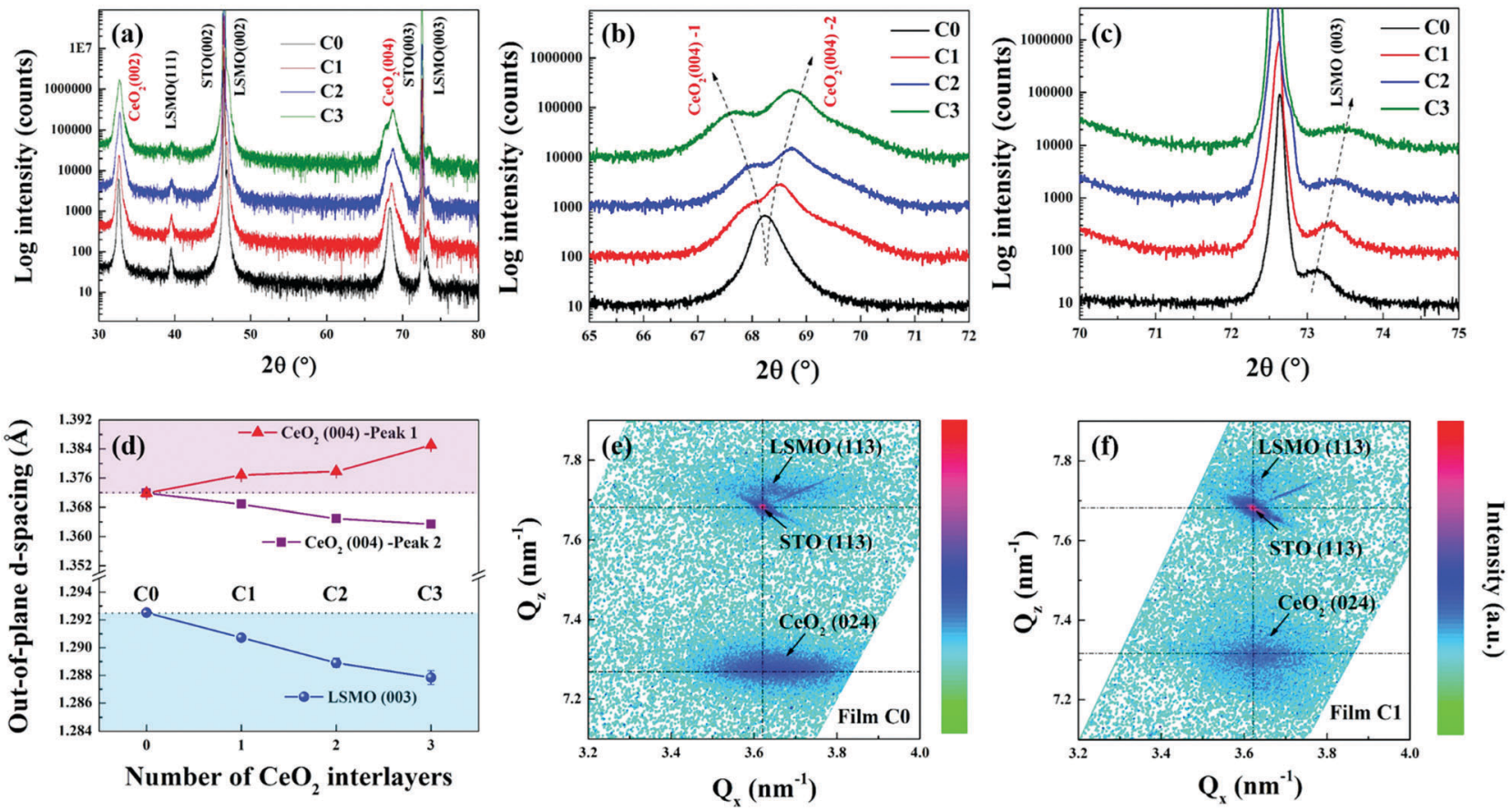

Fig. 3 (a) XRD $2 \theta-\omega$ patterns of the VAN thin film $\mathrm{CO}$ and the $3 \mathrm{D} \mathrm{CeO}_{2}$ framed thin films $\mathrm{C} 1-\mathrm{C} 3$. (b) $\mathrm{Local} \mathrm{CeO}_{2}(004) 2 \theta-\omega$ scans of the VAN thin film $\mathrm{CO}$ and the $3 \mathrm{D} \mathrm{CeO}_{2}$ framed thin films C1-C3. (c) Local LSMO(003) $2 \theta-\omega$ scans of the VAN thin film C0 and the 3D CeO framed thin films C1-C3. (d) Systematic tuning of the out-of-plane $d$-spacing of $\mathrm{CeO}_{2}(004)$ and LSMO(003) by the 3D structure engineering in $\mathrm{CO}-\mathrm{C} 3$ (the $d$-spacing values and error bars are listed in Table S3, ESI $\dagger$ ). The red and purple lines belong to $\mathrm{CeO}_{2}(004)$ Peak 1 and 2, respectively, while the blue line belongs to the LSMO(003) peak. The pink regime represents the tensile out-of-plane strain area of the $\mathrm{CeO}_{2}$ phase and the blue regime represents the compressive out-of-plane strain area of the LSMO phase, compared to sample C0. Reciprocal space maps (RSMs) of (e) the VAN thin film C0 and (f) the 3D framed thin film $\mathrm{C} 1$ near STO(113). The spots of $\mathrm{LSMO}$ and $\mathrm{CeO}_{2}$ in the RSM reveal the high quality epitaxy growth of the two phases on STO substrates.

$\mathrm{CeO}_{2}(004)$ peak starts to split into two peaks, i.e., Peak 1 and 2, which are getting further apart (Fig. 3b). This suggests the coexistence of two different strain states in the $\mathrm{CeO}_{2}$ phase for the cases of $\mathrm{C} 1-\mathrm{C} 3$. To differentiate the contributions of the two $\mathrm{CeO}_{2}$ peaks, the XRD data was carefully examined. As the number of the lateral $\mathrm{CeO}_{2}$ interlayer increases, the intensity and sharpness of Peak 2 (on the right) increases, and Peak 1 (on the left) maintains its similar intensity. It suggests that Peak 2 is attributed to the $\mathrm{CeO}_{2}$ lateral layers and Peak 1 is from the $\mathrm{CeO}_{2}$ vertical nanopillars. The origins of the two $\mathrm{CeO}_{2}(004)$ peaks are further confirmed by a strain mapping using geometric phase analysis (GPA) in Fig. 4, to be discussed later. Simultaneously, the LSMO(003) peak shifts to higher angles as the interlayer number increases (Fig. 3c). According to the XRD patterns and the Bragg's law, the $d_{(00 t)}$-spacing values of both $\mathrm{CeO}_{2}$ and LSMO phases are calculated and plotted in Fig. 3d (also listed in Table S3, ESI $\dagger$ ). In the VAN thin film C0, $d_{\mathrm{CeO}_{2}(004)}$ is $\sim 1.372 \AA$ and $d_{\mathrm{LSMO}(003)}$ is $\sim 1.293 \AA$, which are marked with dashed lines, respectively. In Fig. 3d, the upper branch $\left(\mathrm{CeO}_{2}\right.$ Peak 1) of $d_{\mathrm{CeO}_{2}(004)}$ representing the vertical $\mathrm{CeO}_{2}$ columns gradually increases from $1.377 \AA$ (C1) to $1.385 \AA$ (C3) as the number of $\mathrm{CeO}_{2}$ interlayers increases. The lower branch $\left(\mathrm{CeO}_{2}\right.$ Peak 2) marked in purple represents $d_{\mathrm{CeO}_{2}(004)}$ of the lateral $\mathrm{CeO}_{2}$ interlayers and gradually reduces from $1.369 \AA$ (C1) to $1.363 \AA$ (C3). The out-of-plane strain is calculated and listed in the (Table S4, ESI $\dagger$ ). Compared to the single layer $\mathrm{LSMO}_{-} \mathrm{CeO}_{2}$
VAN sample $\mathrm{C} 0$, the vertical $\mathrm{CeO}_{2}$ nanopillars are in the tensile out-of-plane strain of $0.361 \%$. The lateral $\mathrm{CeO}_{2}$ layer is under the compressive out-of-plane strain of $-0.224 \%$ in $\mathrm{C} 1$. With increasing lateral $\mathrm{CeO}_{2}$ interlayers in $\mathrm{C} 3$, the out-of-plane strain in the $3 \mathrm{D} \mathrm{CeO}_{2}$ framework increases almost three times compared to that of $\mathrm{C} 1$. The vertical $\mathrm{CeO}_{2}$ nanopillars of $\mathrm{C} 3$ are under $0.962 \%$ tensile strain out-of-plane and the lateral $\mathrm{CeO}_{2}$ interlayers are under $-0.618 \%$ compressive out-of-plane. In comparison, from $\mathrm{C} 1$ to $\mathrm{C} 3, d_{\mathrm{LSMO}(003)}$ is reduced from $1.291 \AA$ (C1) to $1.288 \AA$ (C3), corresponding to a gradually enhanced compressive strain out-ofplane from $-0.139 \%$ (C1) to $-0.362 \%$ (C3). Overall, compared to the reference $\mathrm{C} 0$, the out-of-plane strain coupling between the $\mathrm{CeO}_{2}$ vertical nanopillars and LSMO matrix in $\mathrm{C} 1-\mathrm{C} 3$ is obviously strengthened as the number of $\mathrm{CeO}_{2}$ interlayer increases, indicating the effectiveness of the 3D strain framework. Fig. 3e and f compare the reciprocal space maps (RSMs) near the substrate STO(113) peak for $\mathrm{C} 0$ and $\mathrm{C} 1$, respectively. Again, the strain state evolution is further confirmed by the RSM data, i.e., from C0 to C1, the LSMO(113) peak spot is shifted upwards similar to that in Fig. 3c and $\mathrm{CeO}_{2}(024)$ is broadened along $Q_{Z}$ direction reflecting the peak splitting in Fig. 3b. In comparison, the 3D LSMO frameworks (L1-L3) reduce the out-of-plane strain coupling between the $\mathrm{CeO}_{2}$ vertical nanopillars and LSMO matrix with the increasing number of lateral LSMO interlayers (Fig. S5 and Table S5, ESI $\dagger$ ). For these cases, the strain tunability of the epitaxial thin films is mainly determined by the 3D interconnected LSMO frames. 

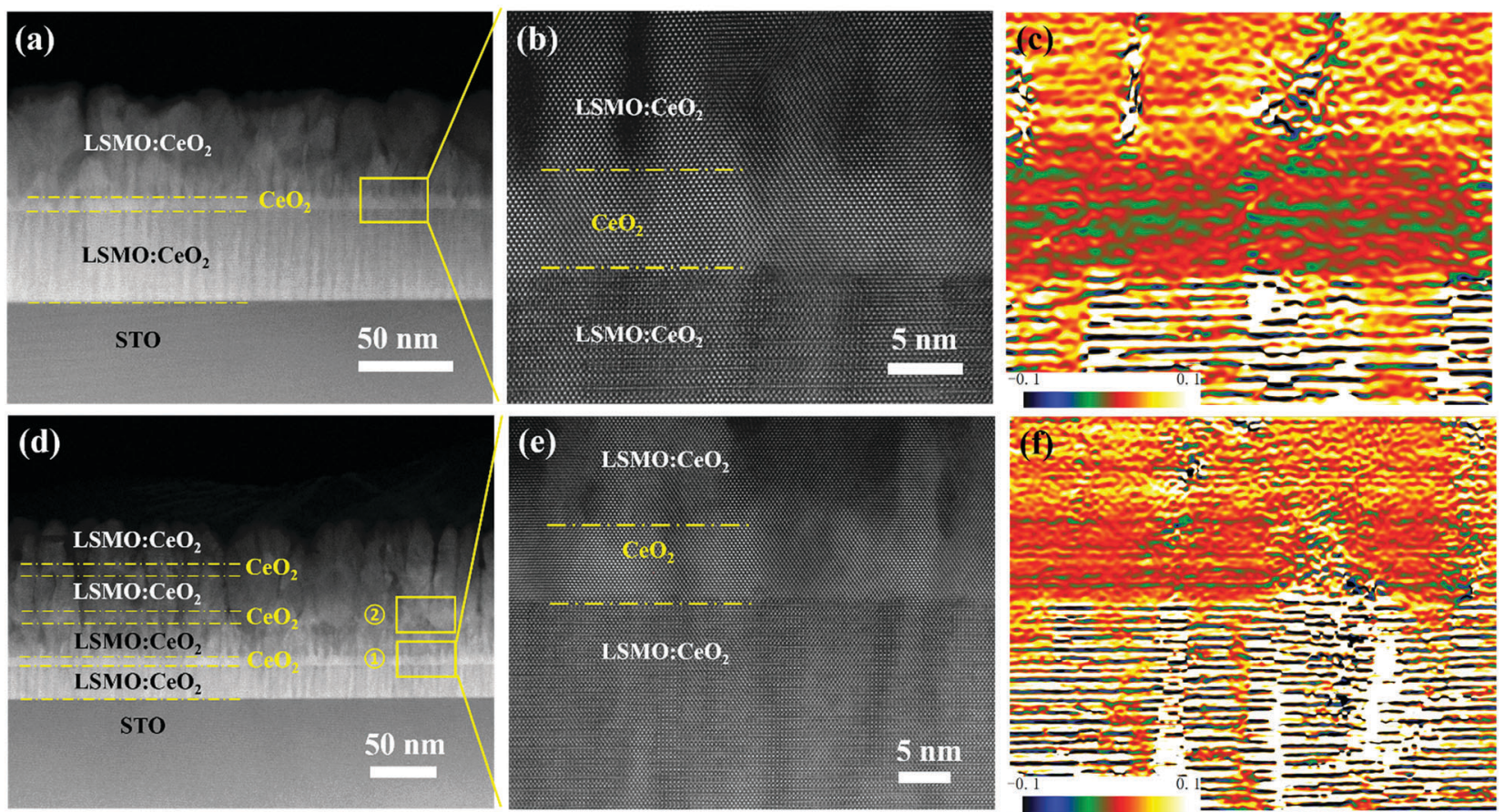

Fig. 4 (a) Cross-sectional STEM image of the $3 \mathrm{D} \mathrm{CeO}_{2}$ framed thin film $\mathrm{C} 1$, (b) its $\mathrm{HRSTEM}$ image at the lateral $\mathrm{CeO}_{2}$ interlayer area (marked by a yellow rectangular in (a)), and (c) the corresponding GPA $\varepsilon_{y y}$ (out-of-plane strain) map of the image shown in (b). (d) Cross-sectional STEM image of the 3D CeO 2 framed thin film C3, (e) its HRSTEM image at the first lateral $\mathrm{CeO}_{2}$ interlayer area from bottom (marked by the yellow rectangular as 1), and (f) the corresponding GPA $\varepsilon_{y y}$ (out-of-plane strain) map of the image shown in (e). Here the lateral $\mathrm{CeO}_{2}$ interlayer area is selected as the reference in each GPA $\varepsilon_{y y}$ map of (c) and (f), and thus exhibits a red-green color. The $\mathrm{CeO}_{2}$ vertical nanopillars within VAN layers are shown in a bright yellow color, illustrating larger out-of-plane $d$-spacing in those vertical $\mathrm{CeO}_{2}$ nanopillars compared to its reference of the lateral $\mathrm{CeO}_{2}$ interlayer area.

Fig. 4a and b exhibit the STEM images of sample C1 under a high-angle-annular-dark-field (HAADF) mode in low magnification and high resolution, respectively. Consistent with the previous TEM results, the STEM images confirm the 3D interconnected $\mathrm{CeO}_{2}$ frames embedded in the films. High resolution STEM images (Fig. 4b) show a very clear interface between $\mathrm{CeO}_{2}$ and LSMO with a high epitaxial quality. A similar structure is observed at the first lateral $\mathrm{CeO}_{2}$ interlayer of sample $\mathrm{C} 3$ (Fig. $4 \mathrm{~d}$ and e). However, as the number of interlayer increases, a large surface roughness is seen. To clearly resolve the $3 \mathrm{D}$ strain state in the samples, geometric phase analysis (GPA) was conducted using the high resolution STEM images (Fig. $4 \mathrm{~b}$ and e), and presented as the vertical strain $\left(\varepsilon_{y y}\right)$ maps in Fig. $4 \mathrm{c}$ and $\mathrm{f}$, respectively. Here the lateral $\mathrm{CeO}_{2}$ interlayer area is selected as the reference in each GPA $\varepsilon_{y y}$ mapping and is shown by a red-green color. The bright yellow color contrast of $\mathrm{CeO}_{2}$ in the VAN part suggests that the vertical $\mathrm{CeO}_{2}$ nanopillars have a larger out-of-plane $d$-spacing, i.e. a tensile strain out-of-plane compared to the lateral $\mathrm{CeO}_{2}$ layer, which is consistent with our observation of the two split peaks in the XRD data (Fig. 3). A sharp change of $\varepsilon_{y y}$ can be clearly seen across the lateral $\mathrm{CeO}_{2}$ and VAN layers, indicating that the $c$-lattice parameter of $\mathrm{CeO}_{2}$ varies abruptly in those layers. It is consistent with its filtered image (Fig. S3, ESI $\dagger$ ), in which the lateral $\mathrm{CeO}_{2}$ interlayer shows a smaller out-of-plane $d$-spacing and the $\mathrm{CeO}_{2}$ in the VAN part presents a larger tensile strain out-of-plane. These observations confirm the distribution of the distinct $d_{00 l}$-spacing within the homogeneous 3D interconnected $\mathrm{CeO}_{2}$ frameworks, i.e., the $d_{00}$-spacing of $\mathrm{CeO}_{2}$ is much higher along the vertical nanopillars than that in the lateral $\mathrm{CeO}_{2}$ layers. Such enhanced outof-plane strain between $\mathrm{CeO}_{2}$ and LSMO is caused by the effective $3 \mathrm{D} \mathrm{CeO}_{2}$ frames and the interfacial coupling between the $\mathrm{CeO}_{2}$ nanopillars and the LSMO matrix. Such vertical strain could potentially lead to the variation of the length and angle of $\mathrm{Mn}^{3+}-\mathrm{O}-\mathrm{Mn}^{4+}$ bonds and thus affect the overall magnetotransport properties, which has been previously reported. ${ }^{12,41,42}$ Overall the finding confirms our initial 3D strain design where a highly strained LSMO can be achieved using a $3 \mathrm{D} \mathrm{CeO}_{2}$ framework.

To resolve the transport properties in these $3 \mathrm{D}$ framed structures, the temperature dependent resistance $(R-T)$ curves at zero-field are shown in Fig. 5a for samples C0-C3. Overall, reducing resistance with increasing temperature represents a typical semiconductor behavior in $\mathrm{C} 0-\mathrm{C} 3$, because of the large portion of $\mathrm{CeO}_{2}$ introduced in the nanocomposites $\left(\mathrm{CeO}_{2}\right.$ : LSMO $\geq 1: 1$ in C0-C3). Fig. $5 b$ plots the temperature dependence of the magnetoresistance (MR\%) in the 3D framed nanocomposite films C0-C3, with clearly defined MR peaks located near $50 \mathrm{~K}$. The MR\% of films C0-C3 increases at first and then reduces as the temperature increases from low temperature to room temperature. The MR\% peak value of the VAN thin film C0 is $\sim 40 \%$, which is higher than many other reported LSMO composite films. ${ }^{27,36,38,43-45} 3 \mathrm{D} \mathrm{CeO}_{2}$ frameworks further enhance the overall MR properties, where the MR peak value increases from $40 \%(\mathrm{C} 0)$ to $51 \%(\mathrm{C} 3), 57 \%$ (C2) and maximizes at $66 \%(\mathrm{C} 1)$. The enhancement can be explained by the $3 \mathrm{D} \mathrm{CeO}_{2}$ 

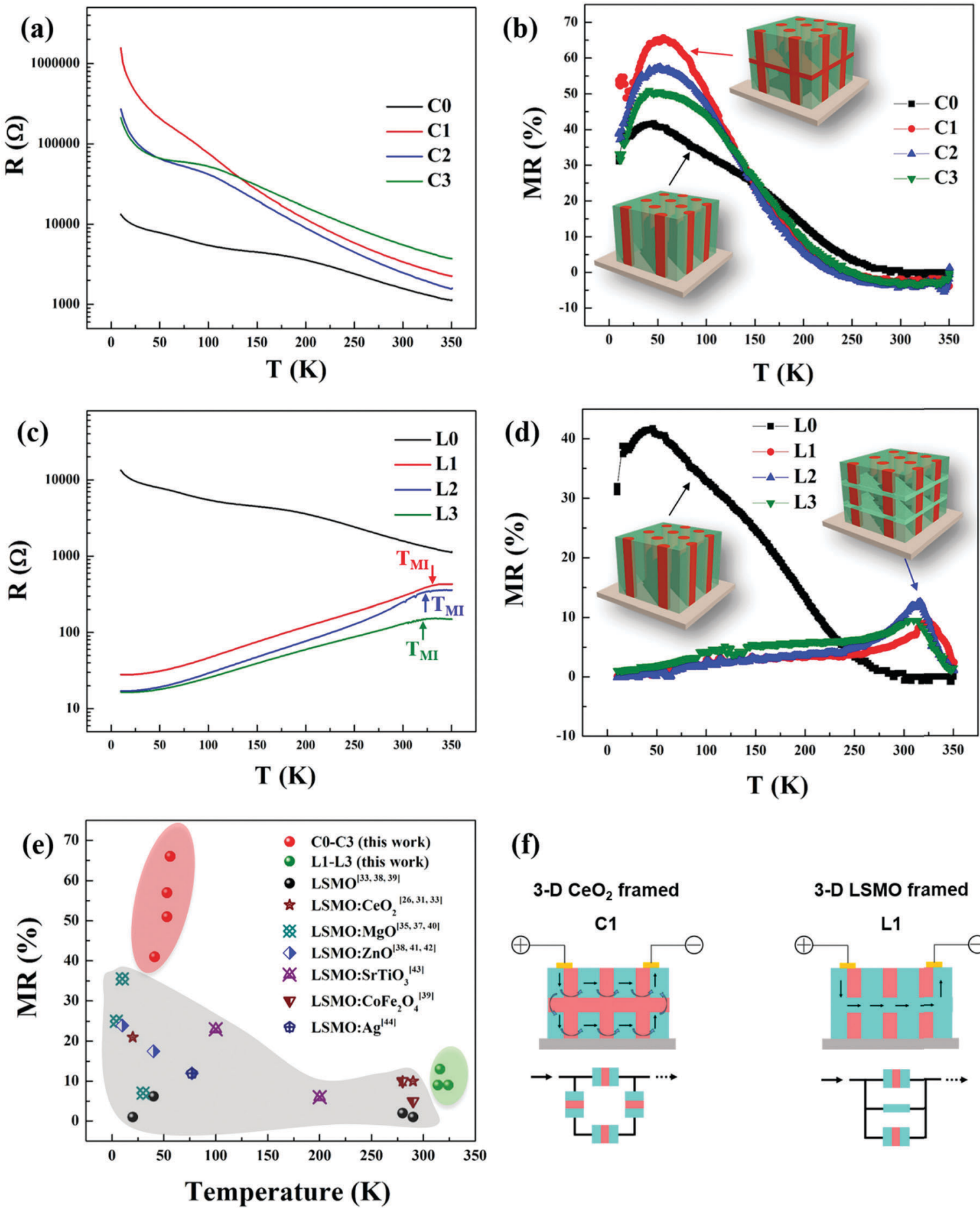

(f)

3-D CeO ${ }_{2}$ framed
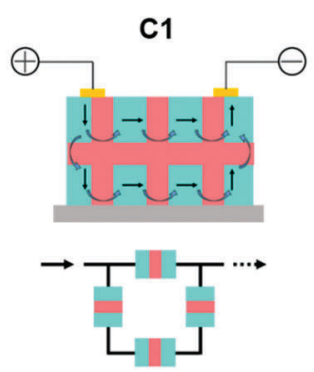

3-D LSMO framed
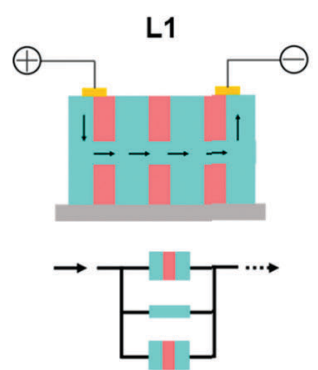

Fig. 5 (a) $R-T$ plots of 3D $\mathrm{CeO}_{2}$ framed nanocomposite thin films $\mathrm{CO}-\mathrm{C} 3$. (b) The temperature dependence of MR for the nanocomposite thin films $\mathrm{CO}-\mathrm{C}$. (c) $R-T$ plots of $3 D$ LSMO framed nanocomposite thin films LO-L3. The arrows point out the metal-to-insulator transition temperature $T_{M I}$ of $L 1-L 3$. (d) The temperature dependence of $M R$ for the nanocomposite thin films LO-L3 with the metal-to-insulator transition temperature $T_{M I}$ marked for samples $L 1-L 3$. (e) Summary of the maximum LFMR values of LSMO-based composite thin films recently reported and their corresponding peak temperature. ${ }^{26,31,33,35,37-44}$ All the LFMR values listed here are measured under the same magnetic field of $1 \mathrm{~T}$ applied out-of-plane (perpendicular to the film plane). (f) Schematic illustrations of the circuit models for the 3D $\mathrm{CeO}_{2}$ framed nanocomposite structure $\mathrm{C1}$ and the 3D LSMO framed nanocomposite film structure L1.

framework as indicated in Fig. 5f. Such 3D $\mathrm{CeO}_{2}$ framework not only tailors the out-of-plane strain of the LSMO phase, but also builds up the 3D tunneling framework for the electron transport. The synergistic effect between the strain tuning and the tunneling effect in C1-C3 (red dots) highly promotes the MR properties and enables a record high MR\% in LSMO nanocomposites (Fig. 5e). ${ }^{26,36,38,43,45-52}$ The relatively lower $\mathrm{MR} \%$ in $\mathrm{C} 2$ and $\mathrm{C} 3$ compared to $\mathrm{C} 1$ is possibly related to the 
surface roughness observed in both samples where the $3 \mathrm{D}$ insulating framework might not be effective in the top layers.

The L1-L3 samples with a 3D LSMO framework exhibit a metallic behavior in contrast to the C1-C3 samples (Fig. 5c), as their resistances gradually increase from $10 \mathrm{~K}$ to $350 \mathrm{~K}$ with a metal-to-insulator transition temperature $T_{\mathrm{MI}}$ at $\sim 325 \mathrm{~K}$. The metallic behavior is associated with the high composition of LSMO in L1-L3 and the 3D interconnected conductive LSMO frames built in the composite films L1-L3. Meanwhile, the resistance of the composite films L1-L3 decreases with inserting more lateral LSMO interlayers over the entire temperature regime. The LSMO interlayers interconnect with the vertical LSMO domains forming a conductive 3D frame in the film. Thus, the tunneling magnetoresistance effect is effectively reduced. But interestingly, such L1-L3 structures enable higher MR values at higher temperatures, e.g. $13 \%$ at $316 \mathrm{~K}$ in sample L2, which is a dramatic MR value improvement compared to $\mathrm{C} 0-\mathrm{C} 3$ and the previous reports at higher temperatures (e.g., near room temperature).

Based on the above observations, it is clear that magnetic tunneling junctions (MTJ) of $\mathrm{LSMO} / \mathrm{CeO}_{2} / \mathrm{LSMO}$ and their geometrical arrangement in these composite films are very important for enhancing the LFMR properties. In C1-C3 samples, there are effective vertical and lateral MTJ structures integrated in the system by incorporating $\mathrm{CeO}_{2}$ interlayers in the VAN system as indicated in Fig. 5f. Such 3D insulating frameworks effectively maximize the 3D magnetic tunneling effect and lead to a record high MR\% in the LSMO based systems.

Most of the previous nanocompositing efforts focus on single layer VAN structures. For example, changing the dimension and density of $\mathrm{MgO}$, Chen et al. altered the strain and magnetic properties of LSMO-MgO VAN systems and tripled the MR value to

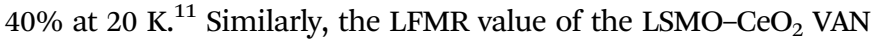
structure was enhanced from $5 \%$ to $21 \%$ at $20 \mathrm{~K}$ by reducing the secondary phase domain size from $\sim 7 \mathrm{~nm}$ to $\sim 3 \mathrm{~nm} .{ }^{38}$ Fan et al. systematically shifted the transition temperature and promoted the LFMR up to $\sim 35 \%$ at $20 \mathrm{~K}$ by tuning the shape and relative ratio of $\mathrm{CeO}_{2}$ domains in the $\mathrm{LSMO}-\mathrm{CeO}_{2}$ VAN system. ${ }^{36}$ This work with a 3D strain framework using both the lateral and vertical interlayers structures effectively maximizes the 3D strain state in the systems, manipulates the electron transport paths in these systems, and thus enables even larger tunabilities of the strainenabled physical properties and the overall electrical transport phenomena. In addition, the 3D framed structure can be realized in other compositions of the VAN systems. As a demonstration, a multilayer stack of $\mathrm{L} 7 \mathrm{C} 3 / \mathrm{CeO}_{2} / \mathrm{L} 7 \mathrm{C} 3$ is constructed to demonstrate the feasibility of the 3D frame designs in other compositions. As shown in Fig. $\mathrm{S} 7$ (ESI $\dagger$ ), the lateral $\mathrm{CeO}_{2}$ interlayer interconnects with vertical aligned $\mathrm{CeO}_{2}$ nanopillars in the VAN L7C3 layers and forms the $3 \mathrm{D} \mathrm{CeO}_{2}$ framed structure, very similar to the case of L5C5. Furthermore, XRD (Fig. S7e and f, ESI $\dagger$ ) and the corresponding SAED patterns (Fig. S7c and d, ESI $\dagger$ ) all demonstrate excellent epitaxial quality of the 3D stack and obvious strain tuning introduced by the $\mathrm{CeO}_{2}$ interlayer. This further confirms the power and feasibility of 3D strain effects generated by this 3D framed microstructure in the epitaxial thin films.

\section{Conclusions}

The 3D framework thin films of $\mathrm{LSMO}^{-\mathrm{CeO}_{2}}$ epitaxial VANs interlayered with either $\mathrm{CeO}_{2}$ or LSMO are designed and processed to effectively control the strain state of the films as well as the electron transport phenomena. This 3D strained framework structures combine both the lateral strain by the layered structures and the vertical strain in the VAN and thus maximize the overall strain in the films. Under the synergistic effects of the 3D maximized strain tuning and the integrated vertical and lateral magnetic tunnel junction structures, the $3 \mathrm{D}$ $\mathrm{CeO}_{2}$ interlayered samples show a record high $\mathrm{MR} \%$ of $51-66 \%$, while the 3D LSMO interlayered thin films boost the MR\% peak in the relatively higher temperature regime (near room temperature). This 3D strain framework concept opens up a new avenue to maximize the film strain beyond the initial critical thickness and can be applied to many other material systems with strain-enabled functionalities beyond magnetotransport properties.

\section{Experimental}

\section{Target preparation}

The targets of pure LSMO, pure $\mathrm{CeO}_{2}$, and composite LSMO$\mathrm{CeO}_{2}$ (molar ratio 1:1 of LSMO and $\mathrm{CeO}_{2}$ ) were prepared via a conventional ceramic sintering process. A stoichiometric mixture of $\mathrm{La}_{2} \mathrm{O}_{3}, \mathrm{MnO}_{2}$, and $\mathrm{SrCO}_{3}$ powders were ground, pressed into a disk, and annealed at $1300{ }^{\circ} \mathrm{C}$ in air for $12 \mathrm{~h}$ to form a pure LSMO target. The same procedure was also employed to synthesize the $\mathrm{CeO}_{2}$ target at $1200{ }^{\circ} \mathrm{C}$ for $6 \mathrm{~h}$ and the $\mathrm{LSMO}-\mathrm{CeO}_{2}$ target at $1300{ }^{\circ} \mathrm{C}$ for $12 \mathrm{~h}$, respectively.

\section{Thin film growth}

The 3D LSMO-CeO ${ }_{2}$ framed nanocomposite films were grown on $\mathrm{SrTiO}_{3}$ (STO)(001) substrates via a pulsed laser deposition (PLD) approach with a $\mathrm{KrF}$ excimer laser $(\lambda=248 \mathrm{~nm})$. Before deposition, the chamber was vacuumed to a base pressure of $1.0 \times 10^{-6}$ Torr $(1$ Torr $=133.3 \mathrm{~Pa})$ or better. During deposition, the substrates were maintained at $750{ }^{\circ} \mathrm{C}$ and the oxygen pressure was controlled at 200 mTorr with a laser frequency of $1 \mathrm{~Hz}$. After deposition, the substrates were cooled down at a cooling rate of $10{ }^{\circ} \mathrm{C} \mathrm{min}{ }^{-1}$ under an oxygen pressure of 200 Torr.

To synthesize the 3D framed nanocomposite films with distinct nanostructures, different numbers of deposition pulses were used and summarized in Tables S1 and S2 (ESI $\dagger$ ), including the VAN thin films $\mathrm{C} 0 / \mathrm{L} 0$, the $3 \mathrm{D} \mathrm{CeO}_{2}$ framed thin films $\mathrm{C} 1-\mathrm{C} 3$, and the 3D LSMO framed thin films L1-L3, respectively. Sample $\mathrm{C0}$ has no lateral $\mathrm{CeO}_{2}$ interlayer which is the typical $\mathrm{LSMO}-\mathrm{CeO}_{2}$ VAN structure. From sample $\mathrm{C} 1$ to $\mathrm{C} 3$, the $\mathrm{CeO}_{2}$ interlayer number gradually increases from 1 to 3 .

\section{Characterization}

The phase and orientation of the thin films were characterized by X-ray diffraction (XRD) ( $2 \theta-\omega$ and $\phi$ scans with $\mathrm{Cu} \mathrm{K} \alpha$ radiation, PANalytical Empyrean system). The reciprocal space mapping was also conducted to analyze the strain and $d$-spacing variations of 
the thin films. Transmission electron microscopy (TEM) images, and selected-area electron diffraction (SAED) patterns were collected on FEI TALOS F200X operated at $200 \mathrm{kV}$. The crosssectional and plan-view samples for TEM analysis were prepared by a standard procedure, including manual grinding polishing, and a final precision ion polishing step (PIPS 691, Gatan, Inc.). The high-resolution scanning TEM (STEM) images were collected on an aberration-corrected FEI Titan microscope equipped with a high brightness Schottky-field emission electron source operated at $300 \mathrm{kV}$. A commercial program, a Digital Micrograph plug-in (DM 1.8.3 package, HRTEM Research Inc.), was used for geometric phase analysis (GPA). The GPA method provides the strain mapping of the entire area based on the atomic spacing variation calculated based on the original STEM images. The out-of-plane strain $\varepsilon_{y y}$ is derived to illustrate the local lattice displacement from the reference lattice. The strain in GPA is the relative value and in this work the lateral $\mathrm{CeO}_{2}$ interlayer area is chosen as the reference, which shows with red-green coloration. Due to $\varepsilon_{y y}=\left(c^{\text {local }}-c^{\text {ref }}\right) / c^{\text {ref }}$, the sign of $\varepsilon_{y y}$ represents a tensile ("+") or compressive ("-") strain in the local lattice compared to the reference. GPA yields a strain map with color contours to illustrate the location of the relative strains. Since the lateral $\mathrm{CeO}_{2}$ interlayer area is selected as the reference area, the GPA $\varepsilon_{y y}$ maps in this work (Fig. 4c, f, and Fig. S4b, ESI $\dagger$ ) are used to analyze the out-of-plane strain distribution of $\mathrm{CeO}_{2}$ phase specifically. Magnetron sputtering and shadow masks were used to deposit $\mathrm{Au}$ contacts for the transport measurements. The magnetotransport properties of the thin films were systematically studied by the Physical Properties Measurement System (PPMS Model 6000, Quantum Design) in a four point probe configuration (in Van der Pauw geometry) with a $1 \mathrm{~T}$ magnetic field applied out-of-plane (perpendicular to the film plane) and the current applied in-plane. Each sample was first cooled from $350 \mathrm{~K}$ down to $10 \mathrm{~K}$ without the applied magnetic field, and then heated up to $350 \mathrm{~K}$ under a magnetic field of $1 \mathrm{~T}$. Electrical resistance was recorded in the temperature range of $10-350 \mathrm{~K}$ with and without the applied magnetic field of $1 \mathrm{~T}$. The corresponding magnetoresistance (MR) was calculated by the following equation:

$$
\operatorname{MR}(\%)=\left[\left(R_{0}-R_{\mathrm{H}}\right) / R_{0}\right] \times 100 \%
$$

where $R_{\mathrm{H}}$ and $R_{0}$ is the electrical resistance with and without the applied magnetic field of $1 \mathrm{~T}$ at the same temperature.

\section{Author contributions}

HW conceived and supervised the project. HW, JMD and XZ discussed the experimental design and project planning. XS deposited the $3 \mathrm{D}$-framed $\mathrm{LSMO}-\mathrm{CeO}_{2}$ thin films, performed $\mathrm{XRD}$ analysis and strain calculation. $\mathrm{JH}$ assisted in film deposition and conducted PPMS measurements. JJ and PL conducted the TEM/STEM and HRTEM/HRSTEM analysis. XS, MF, Han W. carried out the reciprocal space mapping and analyses. XS and QL prepared the TEM samples and the schematics under the supervision of XZ. HW, XS, XZ, and JMD drafted and revised the manuscript.

\section{Conflicts of interest}

There are no conflicts of interest to declare.

\section{Acknowledgements}

This work was funded by the U.S. National Science Foundation (Ceramic Program, DMR-1565822 for high resolution STEM and DMR-1643911 for thin film growth). J. J. and H. W. acknowledge the support of the U.S. Office of Naval Research (Contract number: N00014-16-1-2465). Sandia National Laboratories is a multiprogram laboratory managed and operated by National Technology and Engineering Solutions of Sandia, LLC., a wholly owned subsidiary of Honeywell International, Inc., for the U.S. Department of Energy's National Nuclear Security Administration under contract DE-NA0003525. Part of the atomic resolution STEM work was conducted at the Molecular Foundry, supported by the Office of Science, Office of Basic Energy Sciences, of the U.S. Department of Energy under Contract No. DE-AC02-05CH11231. JLM-D acknowledges funding from EPSRC, grants EP/L011700/1 and $\mathrm{EP} / \mathrm{N} 004272 / 1$.

\section{References}

1 Y. Lu, X. W. Li, G. Q. Gong, G. Xiao, A. Gupta, P. Lecoeur, J. Z. Sun, Y. Y. Wang and V. P. Dravid, Phys. Rev. B: Condens. Matter Mater. Phys., 1996, 54, R8357-R8360.

2 A. S. Bhalla, R. Y. Guo and R. Roy, Mater. Res. Innovations, 2000, 4, 3-26.

3 J. Junquera and P. Ghosez, Nature, 2003, 422, 506-509.

4 S. Jin, T. H. Tiefel, M. Mccormack, R. A. Fastnacht, R. Ramesh and L. H. Chen, Science, 1994, 264, 413-415.

5 Y. Moritomo, A. Asamitsu, H. Kuwahara and Y. Tokura, Nature, 1996, 380, 141-144.

6 E. Dagotto, T. Hotta and A. Moreo, Phys. Rep., 2001, 344, 1-153.

7 Y. Maeno, H. Hashimoto, K. Yoshida, S. Nishizaki, T. Fujita, J. G. Bednorz and F. Lichtenberg, Nature, 1994, 372, 532-534.

8 H. Y. Hwang, S. W. Cheong, N. P. Ong and B. Batlogg, Phys. Rev. Lett., 1996, 77, 2041-2044.

9 M. Uehara, S. Mori, C. H. Chen and S. W. Cheong, Nature, 1999, 399, 560-563.

10 D. G. Schlom, L. Q. Chen, C. J. Fennie, V. Gopalan, D. A. Muller, X. Q. Pan, R. Ramesh and R. Uecker, MRS Bull., 2014, 39, 118-130.

11 A. Chen, J.-M. Hu, P. Lu, T. Yang, W. Zhang, L. Li, T. Ahmed, E. Enriquez, M. Weigand, Q. Su, H. Wang, J.-X. Zhu, J. L. MacManus-Driscoll, L.-Q. Chen, D. Yarotski and Q. Jia, Sci. Adv., 2016, 2, 1-9.

12 J. M. Rondinelli, S. J. May and J. W. Freeland, MRS Bull., 2012, 37, 261-270. 
13 D. G. Schlom, L. Q. Chen, C. B. Eom, K. M. Rabe, S. K. Streiffer and J. M. Triscone, Annu. Rev. Mater. Res., 2007, 37, 589-626.

14 D. Fuchs, E. Arac, C. Pinta, S. Schuppler, R. Schneider and H. V. von Lohneysen, Phys. Rev. B: Condens. Matter Mater. Phys., 2008, 77, 1-8.

15 D. G. Schlom, L.-Q. Chen, X. Pan, A. Schmehl and M. A. Zurbuchen, J. Am. Ceram. Soc., 2008, 91, 2429-2454.

16 K. J. Choi, M. Biegalski, Y. L. Li, A. Sharan, J. Schubert, R. Uecker, P. Reiche, Y. B. Chen, X. Q. Pan, V. Gopalan, L. Q. Chen, D. G. Schlom and C. B. Eom, Science, 2004, 306, 1005-1009.

17 S. A. Harrington, J. Y. Zhai, S. Denev, V. Gopalan, H. Y. Wang, Z. X. Bi, S. A. T. Redfern, S. H. Baek, C. W. Bark, C. B. Eom, Q. X. Jia, M. E. Vickers and J. L. MacManusDriscoll, Nat. Nanotechnol., 2011, 6, 491-495.

18 J. L. MaCmanus-Driscoll, P. Zerrer, H. Y. Wang, H. Yang, J. Yoon, A. Fouchet, R. Yu, M. G. Blamire and Q. X. Jia, Nat. Mater., 2008, 7, 314-320.

19 V. Moshnyaga, B. Damaschke, O. Shapoval, A. Belenchuk, J. Faupel, O. I. Lebedev, J. Verbeeck, G. van Tendeloo, M. Mucksch, V. Tsurkan, R. Tidecks and K. Samwer, Nat. Mater., 2003, 2, 247-252.

20 A. P. Chen, Z. X. Bi, Q. X. Jia, J. L. MacManus-Driscoll and H. Y. Wang, Acta Mater., 2013, 61, 2783-2792.

21 J. L. MacManus-Driscoll, Adv. Funct. Mater., 2010, 20, 2035-2045. 22 W. Zhang, A. Chen, Z. Bi, Q. Jia, J. L. MacManus-Driscoll and H. Wang, Curr. Opin. Solid State Mater. Sci., 2014, 18, 6-18.

23 H. Zheng, J. Wang, S. E. Lofland, Z. Ma, L. MohaddesArdabili, T. Zhao, L. Salamanca-Riba, S. R. Shinde, S. B. Ogale, F. Bai, D. Viehland, Y. Jia, D. G. Schlom, M. Wuttig, A. Roytburd and R. Ramesh, Science, 2004, 303, 661-663.

24 W. R. Zhang, R. Ramesh, J. L. MacManus-Driscoll and H. Y. Wang, MRS Bull., 2015, 40, 736-745.

25 J. Huang, J. L. MacManus-Driscoll and H. Wang, J. Mater. Res., 2017, 32, 4054-4066.

26 M. Fan, B. Zhang, H. Wang, J. Jian, X. Sun, J. Huang, L. Li, X. Zhang and H. Wang, Adv. Mater., 2017, 29, 1606861.

27 A. P. Chen, Z. X. Bi, C. F. Tsai, J. Lee, Q. Su, X. H. Zhang, Q. X. Jia, J. L. MacManus-Driscoll and H. Y. Wang, Adv. Funct. Mater., 2011, 21, 2423-2429.

28 H. Yang, Z. E. Cao, X. Shen, T. Xian, W. J. Feng, J. L. Jiang, Y. C. Feng, Z. Q. Wei and J. F. Dai, J. Appl. Phys., 2009, 106, 104317.

29 J. C. Yang, H. J. Liu and Y. H. Chu, MRS Commun., 2016, 6, 167-181.

30 S. Lee and J. L. MacManus-Driscoll, APL Mater., 2017, 5, 042304.
31 X. Sun, Q. Li, J. C. Jiang and Y. B. Mao, Nanoscale, 2014, 6, 8769-8780.

32 X. Sun, Q. Li, Y. N. Lu and Y. B. Mao, Chem. Commun., 2013, 49, 4456-4458.

33 Z. N. Yu, L. Tetard, L. Zhai and J. Thomas, Energy Environ. Sci., 2015, 8, 702-730.

34 C. W. Cheng and H. J. Fan, Nano Today, 2012, 7, 327-343.

35 Y. C. Qiu, K. Y. Yan, H. Deng and S. H. Yang, Nano Lett., 2012, 12, 407-413.

36 M. Fan, W. R. Zhang, F. Khatkhatay, L. G. Li and H. Y. Wang, J. Appl. Phys., 2015, 118, 065302.

37 X. K. Ning, Z. J. Wang and Z. D. Zhang, Adv. Funct. Mater., 2014, 24, 5393-5401.

38 A. P. Chen, Z. X. Bi, H. Hazariwala, X. H. Zhang, Q. Su, L. Chen, Q. X. Jia, J. L. MacManus-Driscoll and H. Y. Wang, Nanotechnology, 2011, 22, 1-6.

39 O. I. Lebedev, J. Verbeeck, G. Van Tendeloo, O. Shapoval, A. Belenchuk, V. Moshnyaga, B. Damashcke and K. Samwer, Phys. Rev. B: Condens. Matter Mater. Phys., 2002, 66, 104421.

40 X. K. Ning, Z. J. Wang and Z. D. Zhang, Adv. Mater. Interfaces, 2015, 2, 1500302.

41 K. H. Ahn, T. Lookman and A. R. Bishop, Nature, 2004, 428, 401-404.

42 A. J. Millis, Nature, 1998, 392, 147-150.

43 S. A. Koster, V. Moshnyaga, K. Samwer, O. I. Lebedev, G. van Tendeloo, O. Shapoval and A. Belenchuk, Appl. Phys. Lett., 2002, 81, 1648-1650.

44 S. J. Zhu, J. Yuan, B. Y. Zhu, F. C. Zhang, B. Xu, L. X. Cao, X. G. Qiu, B. R. Zhao and P. X. Zhang, Appl. Phys. Lett., 2007, 90, 112502.

45 L. Li, X. Q. Zhang, L. Li, X. F. Zhai and C. G. Zeng, Solid State Commun., 2013, 171, 46-49.

46 A. P. Chen, W. R. Zhang, J. Jian, H. Y. Wang, C. F. Tsai, Q. Su, Q. X. Jia and J. L. MacManus-Driscoll, J. Mater. Res., 2013, 28, 1707-1714.

47 C. H. Yan, Z. G. Xu, T. Zhu, Z. M. Wang, F. X. Cheng, Y. H. Huang and C. S. Liao, J. Appl. Phys., 2000, 87, 5588-5590.

48 M. Staruch, C. Cantoni and M. Jain, Appl. Phys. Lett., 2013, $102,062416$.

49 M. Staruch, H. Y. Gao, P. X. Gao and M. Jain, Adv. Funct. Mater., 2012, 22, 3591-3595.

50 B. S. Kang, H. Wang, J. L. MacManus-Driscoll, Y. Li, Q. X. Jia, I. Mihut and J. B. Betts, Appl. Phys. Lett., 2006, 88, 192514.

51 C. Kwon, Q. X. Jia, Y. Fan, M. F. Hundley, D. W. Reagor, J. Y. Coulter and D. E. Peterson, Appl. Phys. Lett., 1998, 72, 486-488.

52 J. Li, Q. Huang, Z. W. Li, L. P. You, S. Y. Xu and C. K. Ong, J. Appl. Phys., 2001, 89, 7428-7430. 